\section{Laparoscopic Sigmoidectomy for Diverticulitis}

H.-P. Bruch, U.J. Roblick, H. Esnaashari (Hrsg.)

Operation Primer, vol. 2, Heidelberg, Springer, 2008, X + 80 S., 70 farb. Abb., 32,05 EUR

ISBN 978-3-540-78451-7

Diese Serie ermöglicht eine geschickte Führung durch laparoskopische chirurgische Eingriffe und wurde entwickelt, um solche Operationen auf die einfachste Weise darzustellen. Dabei wird besonders auf die Beschreibung der genauen Sequenz des Eingriffs geachtet und ebenso auf die Vorbereitungen, die Technik, den eigentlichen Eingriff, die anatomischen Varietäten sowie die Behandlung von schwierigen Situationen, Komplikationen und Fehlern. Am Ende steht ein knappes, aber ausreichendes Literatur- und Sachverzeichnis. Besonders elegant und überzeugend ist die photographische Führung.

Der zweite Band der Serie gibt einen ausgezeichneten, farbig untermalten Überblick über das laparoskopische Vorgehen bei der Sigmadivertikulitis. Erfahrene Operateure bringen dem interessierten und lernbegierigen Leser diesen Eingriff schrittweise nahe; das Erlernen wird durch hervorragendes Bildmaterial erleichtert und beschleunigt. Am Ende stellt der Leser begeistert fest, dass er sich diesen Eingriff viel schwerer vorgestellt hat.

Das Buch kann daher dem jungen Viszeralchirurgen, der heute über $50 \%$ seiner Eingriffe laparoskopisch vornimmt, ohne Wenn und Aber empfohlen werden. Seine Lektüre wird auch für erfahrene Kollegen nutz- und gewinnbringend sein. Sein Preis ist angemessen.

Karl-Joseph Paquet, Bad Kissingen

\section{Risikomanagement in der operativen Medizin}

\section{J. Enneker, D. Pietrowski, P. Kleine}

Heidelberg, Steinkopff, 2007, 190 S., 49,95 EUR

ISBN 978-3-7985-1737-0

Niemand macht gerne Fehler. Dennoch ereignen sie sich täglich. Besonders unangenehm und folgenreich sind Behandlungsfehler in der Medizin. Sie können sich leicht zur Katastrophe entwickeln. Nach Fehlerursachen, die zu diesem Unglück geführt haben, suchen Mediziner häufig erst dann, wenn es zu einer solchen Katastrophe gekommen ist. So wird meist ein Verursacher gefunden und zur Verantwortung gezogen.
Damit meinen die meisten Mediziner, ihrer Verantwortung Genüge getan zu haben. Inzwischen weiß man allerdings, dass solche Fehler selten auf eine Person zurückzuführen sind, sondern äußere Ursachen häufig für die Fehlerentstehung verantwortlich sind. Somit wäre es viel besser, vorher die Ursachen zu kennen oder nach ihnen zu suchen und damit einen größeren Schaden zu vermeiden. Dass dies möglich ist, zeigen Untersuchungen aus der Hochrisikoindustrie wie z.B. der Luftfahrt oder Kernkraft. Die Herausgeber - verstärkt durch hochqualifizierte Juristen und Versicherungswissenschaftler haben im vorliegenden Werk darstellt, wie Risikovermeidung und -management in der Medizin aussehen sollte. Jedes Kapitel beginnt mit einem humorvollen Leitsatz, ist meist durch eine oder mehrere Abbildungen bzw. Tabellen illustriert und hat häufig ein Literaturverzeichnis. Ergänzt werden die Spezialkapitel durch eine Zusammenfassung der Berichtssysteme aus anderen Ländern, ein Glossar, ein allgemeines Literaturverzeichnis und ein aussagekräftiges Sachverzeichnis. Dieses Buch füllt sicherlich ein Lücke und wird hoffentlich dazu beitragen, in Zukunft Behandlungsfehler zu vermeiden und/oder zu dem richtigen Management nach ihren unbeabsichtigten Auftreten beizutragen. Somit sollte jeder Mediziner, insbesondere aber die operativ Tätigen dieses Buch lesen und zu seiner Verbreitung beitragen. Sein Preis ist angemessen.

Karl-Joseph Paquet, Bad Kissingen

\section{Ärztliche Wundversorgung - Das ABC der Wundheilung}

J.E. Grey, K. Harding (Hrsg.)

München, Urban \& Fischer bei Elsevier, 2008, 110 S., 29,95 EUR

ISBN 978-3-437-24710-1

Die Wunde wurde stets als Ausdruck der Verletzbarkeit der menschlichen Integrität und als äußerlich sichtbare Gefahr für das Leben betrachtet. Bereits in frühgeschichtlichen Zeiten haben sich Menschen mit der Therapie von Wunden beschäftigt. Wundheiler haben Heilungsprinzipien entwickelt, die im Mittelalter, z.b. durch «Feldscher» (Feldchirurgen), weiterentwickelt wurden. Ärzte und Ärztinnen, die sich erfolgreich mit der Wundtherapie befassen, erfreuen sich hoher gesellschaftlicher Anerkennung. Aber auch heute haben noch viele Wundpatienten Probleme, einen Arzt mit Erfahrung in der Wundbehandlung und Interesse an dieser kostenintensiven Problematik zu finden. Trotz technischer Fortschritte in der Chirurgie und Antibiotikaprophylaxe kommt es bei zirka 10\%

\section{KARGER}

Fax +497614520714

Information@Karger.de

www.karger.com (c) 2008 S. Karger GmbH, Freiburg

Accessible online at:

www.karger.com/cga 
der Patienten auch nach einer «sauberen», aseptischen Operation zur Infektion der Operationswunde. Bis zu 15\% der Gesundheitskosten entfallen auf den Diabetes mellitus und seine Folgeerkrankungen, und der größte Teil davon auf das diabetische Fußsyndrom. Letzteres ist die häufigste Ursache für eine stationäre Aufnahme. Darüber hinaus wird zirka 5\% des Gesundheitsbudgets in Deutschland für die Therapie von Dekubitalgeschwüren ausgegeben - und dies, ebenso wie bei diabetischen Fußsyndrom, mit steigender Tendenz. Wunden sind naturgemäß in ihrer Erscheinungsform außerordentlich vielfältig, bedürfen daher der unterschiedlichsten Interventionen und der Behandlung durch ein interdisziplinärer Team. Die Bündelung der Behandlung durch solche Teams mündet so in eine individuelle Therapie für jeden Wundpatienten. Das hier vorgestellte $\mathrm{ABC}$ der Wundheilung soll dem Kliniker einen umfassenden Einblick in das Spektrum der Wundprobleme, wie sie sich Fachleuten darstellt, ermöglichen. Das erste Kapitel beinhaltet die fachgerechte Beurteilung einer Wunde - die entscheidende Grundlage jeglicher Wundversorgung. Die weiteren Kapitel behandeln besondere Arten von Wunden wie chronische und akute Wunden sowie Aspekte der Therapie mit Wundauflagen, Apparaten, Medikamenten, chirurgischen Verfahren und physikalischen, chemischen und biologischen Anwendungen. Die meisten Autoren stammen aus Wales. Dort an der Universität von Cardiff gibt es ein klinisch geführtes Forschungsinstitut, dessen große, akademisch orientierte Forschungsgruppe Kern eines kürzlich eingerichteten «Netzwerks Verbrennungswunden» ist. Für die einzelnen Schwerpunkte dieses Buches sind Autoren verantwortlich, die auf dem jeweiligen Fachgebiet über große Expertise verfügen. Die Beiträge sind in Form von umfassenden Übersichtsartikeln verfasst, in die die jahrelange Erfahrung der Autoren mit einfließt. Ale Beiträge sind hochaktuell, hochqualifiziert und in sehr anschaulicher und didaktisch gelungener Form verfasst und illustriert. Das Buch liefert allen mit der Wundtherapie befassten Ärzten, somit allen operativen Fächern und den nachbehandelnden Ärzten, insbesondere Allgemeinmedizinern und niedergelassenen Allgemein-, Unfall- und plastischen Chirurgen, alles nötige Wissen für die ärztliche Wundversorgung. Es wird daher diesem Leserkreis ohne Einschränkung und mit hoher Penetranz empfohlen. Sein Preis ist günstig und dürfte kein Anschaffungshindernis darstellen.

Karl-Joseph Paquet, Bad Kissingen

\section{Läsionen der Becken- und Oberschenkelarterien}

W. Hepp, A. Gußmann, R.L. Rückert (Hrsg.)

Heidelberg, Steinkopff, 2008, 144 S., 49 Abb., 41 Tab.,

37,95 EUR

ISBN 978-3-7985-1716-5

Die Bände der Berliner Gefäßchirurgischen Reihe stellen eine interessante und auf aktuellen Ergebnissen basierende
Ergänzung der großen gefäßchirurgischen Werke dar. 15 Jahre sind vergangen, seit die aortoiliakalen und femoropoplitealen Arterienverschlüsse in der Berliner Gefäßchirurgischen Reihe zum letzten Mal dargestellt worden sind. Sehr Vieles hat sich seither bei der Behandlung dieser Verschlüsse, aber auch in der gesamten Gefäßchirurgie verändert. Bei der peripheren arteriellen Verschlusskrankheit (pAVK) hat eine Rückbesinnung vom Bypass zur Thrombendarteriektomie stattgefunden, die fast immer intraoperativ mit angiographischer oder endoskopische Kontrolle auf Vollständigkeit vorgenommen wird. Bei der Hybridoperation wird zur Sicherung eines langfristigen Erfolgs - die pAVK ist stets progredient - eine Stent eingesetzt. Alternativ kann vom Radio- oder Angiologen ein solcher Stent nach Angioplastie ohne Operation eingeführt werden. Allerdings müssen nicht selten gefäßchirurgische rekonstruktive Zusatzmaßnahmen in der gleichen, häufiger aber in einer anderen Etage, vorgenommen werden. Daher war der Zeitpunkt für die Darstellung eines aktuellen «State of the art» überfällig. Dieser Band fasst die Ergebnisse des 20. Gefäßchirurgischen Symposiums in Berlin zusammen. Die wesentlichen Aspekte und Fortschritte der Thematik werden abgehandelt. Stellung genommen wird auch zum minimal invasiven Zugang. Ein Schwerpunktthema ist das Beckenarterienaneurysma, das gerade im Rupturstadium viel gefährlicher als das infrarenale Aortenaneurysma ist. Im Schwerpunkt Oberschenkelarterien wird unter anderem über erste Erfahrungen mit der Dynaflo-Prothese im femoropoplitealen Bereich berichtet. Interessant ist sicher auch die Frage, was sich in 50 Jahren Bypass-Chirurgie geändert hat. Die einzelnen Vorträge sind interessant, informativ, didaktisch gut dargeboten und ausreichend illustriert. Die Lektüre diese Bands wird daher das Wissen angehender und auch erfahrenen Gefäßchirurgen bereichern. Auch Angiologen und Radiologen, die zur interdisziplinären Kooperation mit Gefäßchirurgen bereit sind dies sollte eigentlich selbstverständlich sein -, können beim Lesen dieses Buchs profitieren. Sein Preis ist günstig.

Karl-Joseph Paquet, Bad Kissingen

\section{Langenscheidt Arzt - Deutsch Deutsch - Arzt}

\section{E. von Hirschhausen}

Berlin, Langenscheidt, 2007, 128 S., 9,95 EUR

ISBN 978-3-468-73177-8

Der in Frankfurt wohnende Mediziner und Wissenschaftsjournalist, der seit 15 Jahren als Kabarettist, Humortrainer, Redner und Autor präsent und dessen Markenzeichen der intelligente Witz mit nachhaltiger Botschaft ist, hat in handlichen Kleinformat ein Wörterbuch zum Verständnis der medizinischen Fachsprache und des unverständlichen Jargons der Ärzte verfasst. Als Kabarettist hat er dies mit viel hinterhältigem Humor und großer Bravour getan. Er vermittelt dem Laien die wichtigsten Vokabeln der ärztlichen Fachsprache. 
Der ist so in wenigen Stunden in der Lage zu verstehen, was der Arzt sagt, und insbesondere zu begreifen, wenn der Arzt eigentlich nichts sagt, und kann mit neuen Ausdrücken im Wartezimmer, Fitnessstudio und zu Hause Eindruck schinden. Darüber hinaus erklärt das Buch nicht nur Wörter der Fachsprache, sondern auch die wichtigsten nonverbalen Signale wie Kittel, Wartezeiten und disziplinarische Abläufe. Die Erläuterungen sind nicht immer wörtlich, sondern sinngemäß, manchmal auch unsinngemäß zu nehmen; das wird der interessierte Leser schnell herausfinden. Das Büchlein gliedert sich in die Kapitel Symptomcheck zu Hause, Grundkurs Arztsprache, Beim Hausarzt, Untersuchungen, Fachärzte, Halbgötter, Halbengel und Halbstarke, Apotheke und Beipackzettel und wird ergänzt durch ein kurzes Vor- und Nachwort. Ein Sachverzeichnis, das vielleicht bei der Kürze des Büchleins auch entbehrlich ist, gibt es nicht. Die Lektüre dieses Büchleins, das äußerst humorvoll geschrieben und mit farbigen Cartoons gut illustriert ist, besticht zusätzlich durch eine didaktisch geschickte Darstellung mit Merksätzen und durch farbige Kästchen hervorgehobenen Hinweisen. Die Lektüre dieses gelungenen kleinen Buchs kann daher jedem jetzigen und zukünftigen Patienten zur Erweiterung seines Wissens um die Medizin und zum Abbau seiner Frustration gegenüber den fachsprachlich fixierten Ärzten ohne Einschränkung empfohlen werden. Karl-Joseph Paquet, Bad Kissingen

\section{Appendizitis und Differenzialdiagnosen, DVD}

\author{
A. Hollerweger, K. Dirks (Hrsg.) \\ Köln, Deutscher Ärzte-Verlag, 2008, 49,95 EUR \\ ISBN 978-3-7691-0560-5
}

«Appendektomie oder nicht?» ist eine häufige Frage im allgemein- und viszeralchirurgischen Krankenhaus- und Praxisalltag. Auch für Internisten, Radiologen, Allgemeinmediziner und Pädiater ist sie eine wichtige, fast tägliche vorkommende Fragestellung. Die Herausgeber haben es daher unternommen, eine DVD mit den entscheidenden sonographischen Bildern zur Appendizitis und ihren Differentialdiagnosen zusammenzustellen. Zweifellos ist die Ultraschalldiagnostik einer der wesentlichsten Fortschritte der letzten Jahrzehnte bei der Diagnostik der Appendizitis. Sie ist heute Methode der Wahl und wird zunehmend auch von juristischer Seite gefordert. Anhand eines einmaligen Bild- und Videomaterials führt das interdisziplinäre Autorenteam dieser interaktiven DVD Schritt für Schritt in die Thematik ein, beginnend bei den Grundlagen bis hin zu den Tricks und Tipps der Experten. Die DVD hilft, eine Appendizitis rasch und zweifelsfrei zu diagnostizieren und von den möglichen Differentialdiagnosen $\mathrm{zu}$ unterscheiden. So lassen sich unnötige Operationen vermeiden. Ingesamt findet der Leser bzw. Zuschauer eine interaktive Fallsammlung mit über 2100 Einzelbildern und 86 Vi- deoanimationen. Eine farbliche Abgrenzung anatomischer Strukturen erfolgt auf Knopfdruck. Weder fehlen die erläuternden Texte noch am Ende ein ausführliches Literaturverzeichnis. Die wichtigsten Kapitel sind Anatomie und Untersuchungstechnik, Akute Appendizitis, Perforation und Komplikationen, Postoperative Befunde, Pitfalls und Differentialdiagnose. Vor dem Literaturverzeichnis wird auch der Wert des Kernspins und der CT am Rande erwähnt und herausgestellt, dass heute Röntgenaufnahmen (Abdomen leer) und auch Endoskopie - mit nur wenigen Ausnahmen - obsolet sind. Die Lektüre und Bildschau dieser DVD eignet sich für Internisten, Gastroenterologen, Radiologen, Pädiater, Allgemein- und Viszeralchirurgen. Auch Allgemeinmediziner können davon profitieren. Der Preis ist angemessen.

Karl-Joseph Paquet, Bad Kissingen

\section{Mammakarzinom - Operative Behandlungskonzepte}

\section{R. Jakesz, M. Frey (Hrsg.)}

Wien, Springer, 2007, IX + 239 S. 150 Abb., 169,95 EUR

ISBN 978-3-211-29683-7

Von den gleichen Herausgebern ist bereits vor 8 Jahren ein Buch mit dem gleichen Titel erschienen. Das jetzige Werk ist keine überarbeitete 2. Auflage, sondern wurde als Neuauflage konzipiert. Das Mammakarzinom ist die häufigste Krebserkrankung der Frau. Beträchtliche chirurgische Fortschritte der letzten Jahre verbesserten sowohl die Prognose als auch die kosmetischen Ergebnisse. Gerade die rasche Weiterentwicklung der diagnostischen und therapeutischen Disziplinen legen beim Mammakarzinom eine Zentralisierung in Kompetenzzentren nahe, die neben der Optimierung der Behandlung der einzelnen Patientin zur frühen und systematischen Etablierung modernster Standards führt und Grundlage einer fortlaufenden Qualitätskontrolle ist. Beide Autoren arbeiten in einem solchen Zentrum, haben ein solches aufgebaut bzw. befinden sich in einem solchen Aufbau. Am Buchanfang gehen die Autoren kurz auf anatomische Grundlagen ein und geben einen Überblick über derzeit gängige interventionelle, resektive und onkoplastische Konzepte. Etablierte Therapien ergänzen sie zum Teil durch noch experimentelle operative und strategische Modalitäten. Neben der Beschreibung der adäquaten Techniken der operativen Tumorentfernung - abhängig von Faktoren wie Histologie und Ausbreitung des Tumors, Alter und Disposition der Patientin - ist den Autoren vor allem die Vermittlung einer Grundhaltung bei der Erstellung des Behandlungskonzepts wichtig. Deshalb haben sie neben onkologischen operativen Verfahren den Schwerpunkt ihrer Darstellung auf die exakte präoperative Diagnosestellung, auf die besonderen Schwierigkeiten bei Präkanzerosen und In-situ-Karzinomen, auf die Auslotung der Brusterhaltung gegenüber der Mastektomie, auf Diskussionen des operativen Vorgehens beim familiären Brustkrebs, auf die Verein- 
barkeit von Sicherheit und Schonung bei der axillären Lymphadenektomie und schließlich auf die immer mehr an Bedeutung gewinnende präoperative Chemotherapie sowie die darauf abgestimmten Verfahren gelegt. Einerseits beschreiben sie etablierte Standards und Qualitätsnormen, andererseits sind das chirurgische Wissen und darauf aufbauenden Behandlungsstrategien ständig im Fluss. Deshalb liegt der Schwerdenzbasiert Bewährte hinausgehen. Ingesamt erfährt der kritische Leser eine große Bereicherung bei der Lektüre dieses Buchs, das als Neuauflage rundherum gelungen ist. Es wird besonders Chirurgen, Gynäkologen, Onkologen und Strahlentherapeuten zum Studium empfohlen. Auch der Allgemeinmediziner, der häufig die Nachbehandlung solcher Patienten übernimmt, kann von seiner Lektüre profitieren. Sein Preis ist angemessen.

Karl-Joseph Paquet, Bad Kissingen punkt des Buches auf denjenigen Themen, die über das evi-

und exzellentes Bildmaterial gefördert wird. So werden die Prozeduren Schritt für Schritt dargestellt. Das Buch bietet ausführliche Handlungsanweisungen für Effizienz und Sicherheit im Arbeitsalltag und für die optimale Patientenversorgung. Hervorzuheben ist besonders die gute Didaktik bei der Wissensvermittlung und die hervorragenden Illustrationen. Es ist ein interdisziplinäres Buch und spiegelt damit wider, wie heute solche Abteilungen in gastroenterologischen und viszeralchirurgischen Kliniken schon allein aus Kostengründen sein sollten. Es kann daher für Gastroenterologen und insbesondere für Viszeralchirurgen ohne wesentliche Einschränkung zur Anschaffung empfohlen werden. Auch andere Disziplinen werden von seiner Lektüre profitieren. Sein Preis ist angemessen bis günstig.

Karl-Joseph Paquet, Bad Kissingen

\section{Atlas der Brustchirurgie}

\section{Interventionelle Endoskopie - Lehrbuch und Atlas}

\section{S. Kahl, G.F.B.A. Kähler, A.J. Dormann (Hrsg.)}

München, Urban \& Fischer bei Elsevier, 2007, 432 S., 400 farb. Abb., 47 farb. Tab., 209,- EUR

ISBN 978-3-437-23620-4

Die diagnostische und therapeutische Endoskopie übt auf die Phantasie der Menschen eine große Faszination aus. Sie ist in den letzten Jahrzehnten zu wichtigsten Methode für die Erkennung und auch für die Behandlung von Magen-Darm-Erkrankungen geworden und hat sich längst von der bloß betrachtenden Diagnostik zur einer wenig invasiven therapeutischen Option entwickelt. Schließlich sind in den letzten Jahren enorme Fortschritte gemacht worden. Neue Färbetechniken, Muskosaresektionstechniken, Stent-Verfahren usw. ersetzten zunehmend früher notwendige, offene chirurgische Eingriffe. Die Herausgeber, zwei Gastroenterologen und ein Viszeralchirurg, verstärkt durch 34 Autoren aus den Bereichen Gastroenterologie, Viszeralchirurgie, Pathologie, Rechtsmedizin, Medizinische Forschung und Medizinisches Controlling sowie aus Enddarmzentren haben es unternommen, das gesamte Gebiet zu bearbeiten und vorzustellen.. Dieser Versuch kann mit einigen Einschränkungen durchaus als gelungen bezeichnet werden. Teil 1, Gastroenterologische Erkrankungen, ist aufgeteilt nach den Abschnitten des Verdauungssystems stellt anhand einzelner Krankheitsbilder dar, welche diagnostischen und therapeutischen Techniken in Frage kommen und welche Besonderheiten bei der Durchführung und Nachbehandlung zu beachten sind. In Teil 2, Techniken, werden alle interventionellen Verfahren erläutert, die in unterschiedlichen Abschnitten des Verdauungssystems zu Anwendung kommen. Das Ziel des Buchs ist die unmittelbare praktische Umsetzbarkeit der Informationen, was durch hoch strukturierte, knappe Texte

\author{
M. Kaufmann, I. Jatoi, J.Y. Petit (Hrsg.) \\ Heidelberg, Springer, 2008, VIII + 128 S., 291 Abb., \\ 129,95 EUR \\ ISBN: 978-3-540-48881-1
}

Die deutschsprachige Ausgabe des international erfolgreichen «Atlas of Breast Surgery»- herausgegeben von den weltbesten Chirurgen auf diesem Gebiet - ist reichhaltig und anschaulich illustriert. Die Herausgeber berücksichtigen die neuesten Entwicklungen und informieren von der Anatomie über das diagnostische Vorgehen bis hin zur effektiven Behandlung. Patientinnen mit Brusterkrankungen erwarten nicht nur qualifizierte medizinische Betreuung, sondern auch ansprechende ästhetische Ergebnisse. Deshalb liegt der Schwerpunkt auf Techniken, die ein ansprechendes kosmetisches Resultat erwarten lassen. Die onkologisch-chirurgischen sowie plastisch-kosmetischen Verfahren werden geschickt ausgewählt präsentiert. Viele anschauliche Abbildungen zeigen die entscheidenden Schritte des chirurgischen Vorgehens. Leider werden die zahlreichen Abbildungen nur in Schwarz-Weiß präsentiert. Die vorliegende erweiterte Übersetzung der internationalen Ausgabe ist speziell an die Bedürfnisse des deutschsprachigen Europas angepasst und berücksichtigt alle wichtigen Verfahren und Operationsmethoden aus diesem Bereich. Es wurde geschrieben für chirurgisch tätige Gynäkologen, dürfte aber genauso den ästhestisch-plastischen Chirurgen interessieren. Beiden Berufsgruppen wird dieser Atlas daher besonders empfohlen. Sein Preis ist - trotz der zahlreichen, allerdings nur in Schwarz-Weiß vorgestellten Abbildungen - etwas überdimensioniert, zumal es sich vorwiegend um einen erweiterten Extrakt aus einem bereits vorhandenen Werk handelt. Diese Tatsache wird seine Verkaufschancen limitieren.

Karl-Joseph Paquet, Bad Kissingen 


\section{Checkliste Doppler- und Duplexsonographie}

H. Kopp, M. Ludwig

Stuttgart, Thieme, 2007, 3. Aufl., 345 S., 44,95 EUR

ISBN 978-3-13-110933-0

Die Verfahren der Doppler- und Duplexsonograpie sind trotz neuerer Entwicklungen wie der MR- und CT-Angiographie wertvolle und unverzichtbare Werkzeuge für die angiologische Diagnostik geblieben. Ihre Bedeutung hat eher zugenommen. Sie liefern nämlich nicht nur entscheidende Informationen über Lokalisation und Morphologie von Gefäßveränderungen, sondern auch über ihre hämodynamische Relevanz und bestimmen damit Vorgehen, Indikation und Therapiemodalität bei den verschiedenen Krankheitsprozessen. Für die gründliche und umfassende Besprechung dieser Thematik (besonders auch in Form einer Checkliste) sind die beiden Autoren hervorragend geeignet. In der Checkliste Doppler- und Duplexsonographie erhält der Anfänger zunächst exakte Hinweise zur Geräteeinstellung und -bedienung und zur Durchführung jeder einzelnen Untersuchung. Ist sein Wissenstand fortgeschritten, so findet er in dieser Checkliste ebenso seltene Krankheitsbilder, z.B. die Mesenterialarterienstenose, die heute durchaus zuverlässig mit den beschriebenen bildgebenden Verfahren diagnostiziert werden kann. Da gerade diese Erkrankung eine häufige Differentialdiagnose aufweist, eignet sich dieses nichtinvasive und überall durchführbare Verfahren durchaus zum häufigen Einsatz. Wie in den vorausgegangenen Ausgaben werden die häufigsten Fehlerquellen und die wichtigsten Fallstricke analysiert und einer gut verständlichen Beseitigung bzw. Lösung zugeführt. Nicht nur in der speziellen Diagnostik ist die Checkliste unverzichtbar, sondern sie liefert auch viele Hinweise zur weiterführenden Diagnostik und zum therapeutischen Vorgehen. Wie in der Vergangenheit ist das Werk in einen grauen, einen grünen und einen blauen Teil (Grundlagen, Flussschemata und Fallstricke sowie spezielle Gefäßregionen) gegliedert. Am Ende folgt ein Anhang mit Befundungskriterien und Fallbeispielen, ein umfangreiches Sachverzeichnis, ein Kurzglossar und eine Tabelle mit den Normalwerten der Strömungsgeschwindigkeiten. Insgesamt wurde das Buch fachkundig und vollständig überarbeitet und präsentiert den aktuellen Stand des Wissens. Einige Kapitel, wie z.B. die Kontrastmittelsonographie und das Tissue Harmonic Imaging sind hinzugekommen. Auch die Fallbeispiele sind größtenteils neu. Die Lektüre dieser Checkliste kann somit allen Internisten, Gefäßchirurgen und Angiologen zum intensiven Studium und auch als Nachschlagwerk bei unklaren Befunden und ungeklärten Fragestellungen eingehend empfohlen werden. Auch der besonders interessierte Allgemeinmediziner wird von der Lektüre dieses Werkes profitieren. Sein Preis ist - wie bei diesen Checklisten üblich durchaus als günstig zu bezeichnen.

Karl-Joseph Paquet, Bad Kissingen

\section{Breitner Chirurgische Operationslehre, Band 1-14, DVD}

\section{R. Obermeier}

München, Urban \& Fischer bei Elsevier, 2008, 99,95 EUR

ISBN 978-3-437-24830-6

Professor Breitner, Innsbruck war nicht der Erste, der erkannt hatte, welche Bedeutung die Weitergabe von Erfahrung in immer schneller wachsenden und größer werdenden Fach Chirurgie hat und wie wichtig es damals war und heute genauso ist, eine anatomisch korrekte, technisch ausgereifte und konzeptionell logische Operationsplanung durchzuführen. Breitner erstellte aber mit wenigen Mitarbeitern das erste Standardwerk der Chirurgischen Operationslehre; er legte damit den Grundstein für die Zukunft für fast 100 Jahre. Heute sind junge Chirurgen vor allem am Bild im Internet interessiert, vielleicht auch ärmer an Geld, vor allem aber an Zeit und dennoch begeistert für dieses Fach. Sie mussten bisher vor Ersteingriffen den Operationsatlanten in der Bibliothek ausleihen, ihn sich bei ihrem Chef oder erfahrenen Kollegen borgen oder für viel Geld alle 14 Bände der Operationslehre kaufen. Es ist daher dem Verlag sehr zu danken, dass er für alle jungen Chirurgen eine Alternative zu einem sehr attraktiven Preis anbietet; es gibt alle 14 Bände handlich auf einer DVD mit der gesamten Chirurgie in über 5000 hervorragenden Zeichnungen und Illustrationen, mit maximalen Komfort durch übersichtliche Menüführung mit Suchfunktionen und Volltextsuche für leichteres Arbeiten. Die zahlreichen Abbildungen sind zwei- und dreifarbig. Außerdem kann der Leser alle Seiten ausdrucken oder einzelne Elemente ausschneiden. Zwar stammen alle Ausgaben vom Ende der 1980er und Beginn der 1990er Jahre, jedoch sind sie, was die meisten Operationen angeht, auf dem neuesten Stand; dies gilt teilweise auch für minimal invasive Eingriffe. Nur wenige Operationen werden nicht beschrieben, da sie neu entwickelt wurden bzw. noch nicht akzeptiert waren. Dies gilt vor allem für laparoskopisches Arbeiten. Trotz dieser Einschränkung wird dem jungen Allgemein-, Unfall-, Gefäß- Viszeral- und Plastischem Chirurgen ein unglaublich preiswertes Werk von hohem Standard und guter Ausstattung geboten, das daher ohne Einschränkung empfohlen werden kann.

Karl-Joseph Paquet, Bad Kissingen 